\title{
Polytopes vibrations within Coxeter group symmetries
}

\author{
Goce Chadzitaskos $^{1}$, Jiř́ Patera ${ }^{2}$, and Marzena Szajewska ${ }^{3, a}$ \\ 1 Department of physics, Faculty of nuclear sciences and physical engineering, Czech Technical University in Prague, Břehová 7, \\ 11519 Prague 1, Czech Republic \\ 2 Centre de recherches mathématiques, Université de Montréal, C.P. 6128, succ. Centre-ville, Montréal, H3C 3J7, Canada \\ 3 Institute of Mathematics, University of Bialystok, 1M Ciolkowskiego, 15-245 Bialystok, Poland
}

Received 16 November 2015 / Received in final form 11 April 2016

Published online 23 May 2016

(C) The Author(s) 2016. This article is published with open access at Springerlink.com

\begin{abstract}
We are considering polytopes with exact reflection symmetry group $\mathfrak{G}$ in the real 3-dimensional Euclidean space $\mathbb{R}^{3}$. By changing one simple element of the polytope (position of one vertex or length of an edge), one can retain the exact symmetry of the polytope by simultaneously changing other corresponding elements of the polytope. A simple method of using the symmetry of polytopes in order to determine several resonant frequencies is presented. Knowledge of these frequencies, or at least their ratios can be used for control of some principal changes of the polytopes.
\end{abstract}

\section{Physical introduction}

The process, which we want to consider in the paper, involves changing the positions of the vertices and edges of a given polytope $\mathfrak{P}$ while preserving its original symmetry. Necessarily, even the change of one quantity (position or distance on the shell) requires other modifications of the shell of the polytope if the symmetry is to be preserved. Viewing it as a dynamical process, if the change occurs periodically, then it will lead to the vibration of other elements on the polytope shell causing the vibration of the whole polytope structure [1,2]. Describing some of the vibrations is the main goal of the paper.

The possibility of deforming the polytopes by changing the position of vertices raises the question of whether we can exploit the dynamical properties of the polytopes [3]. Taking the displacement of vertices as generalized coordinates in Lagrange mechanics, corresponding oscillations and movements can be found. We will use Hooke's Law approximations for isotropic materials, which holds at least for small amplitudes so that the atomic bond forces can be described spring forces. Using the polytope structures described in $[4,5]$, we discuss below three reasons to analyze the dynamical properties of polytopes.

The first reason for analyzing polytope properties is to allow us to determine the presence of polytopes in a substance. Using polytope vibration frequency modes we can determine quantitatively and qualitatively the presence of polytopes in a material.

\footnotetext{
a e-mail: m.szajewska@math.uwb.edu.pl
}

Analysis of the frequencies identifies the components of the substance. Analysis of the amplitudes determines the relative amount of components.

The second reason for analyzing polytopes is that it enables us to determine the tension of atomic bonds, i.e. molecular forces between atoms in a polytope. This follows from the fact, that in all described cases we determine the frequencies of four modes, and all of them are perpendicular to the square root of their tensions. From spectral analysis one can identify the frequencies once we know also the ratio. The ratio of resonant frequencies can be found using the parameters like masses, number of vertices and number of edges.

The third reason is the possibility to use these frequencies to control the transition between polytopes or their destruction. These frequencies are resonant frequencies. It means that at these frequencies small periodic oscillations cause large amplitudes and increase the kinetic energy. These properties can be used for example for controlling some behaviours of viruses of polytope shape [6].

The description of the transition between some polytopes was shown in $[4,5]$ using several parameters. This enables us to find the vibration modes connected to these parameters. The polytopes generally have $N$ modes, where $N$ is the degree of freedom.

The calculation of all modes and describing the corresponding movements is complicated, because of their interdependence and because of a great number of variables. It prevents us to find the exact solution.

There are some modes, which can be exactly calculated using the symmetry of the polytope. These modes determine the oscillations that can be visualized. The proper 
manipulations of polytopes connected to the modes can be used for their transitions. Moreover, the knowledge of frequencies of modes can help to find the ratio of polytopes of different type in the mixture of polytopes in a material. In order to find the composition of a material one can identify varies polytopes by either their frequencies or by ratios of their frequencies (in some cases the ratios are unique).

The symmetry also plays a role in the determination of the velocities of mass points at the vertices.

The symmetry which we used guarantees that the velocities of all points of polytopes are the same at each moment. The positions of atoms obey the symmetry group and the calculations of tension of atomic bonds is straightforward.

There is an additional mode connected to central symmetry, which is described by the radial vibration of a polytope. The resonant frequencies depend on the ratio of tension and mass.

\section{Construction of polytopes}

In the paper $\mathfrak{G}$ stands for the Weyl groups of the simple Lie groups of rank 3 , namely $A_{3}, B_{3}, C_{3}$, and also the non-crystallographic Coxeter group $H_{3}$ of order 120 (the icosahedral group). All the four groups are acting in the 3 -dimensional real Euclidean space $\mathbb{R}^{3}$.

The simplest polytopes $\mathfrak{P}$, generated by $\mathfrak{G}$, are shells of points/vertices that are equidistant from the origin of $\mathbb{R}^{3}$. All its vertices are generated from any one of them by repeated application of the reflections $r_{1}, r_{2}, r_{3}$ that generate all the elements of $\mathfrak{G}$ [7]. For each of the four groups the reflection mirrors have different relative angles.

We say that the simplest polytopes have vertices from one orbit of $\mathfrak{G}$. The radius of the shell carrying vertices of such polytopes does not enter into the calculations. If however, more than one orbit of $\mathfrak{G}$ should be used in forming the vertices of the polytope $\mathfrak{P}$, the relative length of their radii is of importance.

If more than one orbit of $\mathfrak{G}$ constitute the points of the polytope, two complications of the structure may arise. The first one is the presence of greater number of vertices on the outer shell of the polytope. The second complication would have more points of the polytope present in the interior of the outer shell.

In all cases we assume that the exact symmetry of $\mathfrak{G}$ is preserved. That assumption allows us to limit our consideration to a smaller number of cases that appear to be most interesting at present.

The single shell/orbit polytopes $\mathfrak{P}$ are constructed by repeated reflections $r_{1}, r_{2}, r_{3}$, applied to any one of its points/vertices [7].

\section{Vibrations of $A_{3}$ polytope}

In this symmetry case we illustrate the method, which is then used in the other cases as well. The case is differ in the
Lagrangians and therefore in resulting movements, i.e. the frequencies of modes and the corresponding movements of polytopes.

The generic $A_{3}$ polytope consists of 24 vertices, 12 edges $a, 12$ edges $b$ and 12 edges $c$.

Cartesian coordinates of tetrahedral polytope are expressed in terms of three variables $a, b$ and $c$, which represent the lengths of three types of edges connecting the masses of atoms $m$ at the vertices

$$
\vec{x}=\left(-\frac{a}{2}-\frac{2 b}{3}-\frac{5 c}{6},-\frac{a}{2}-\frac{2 b}{3}+\frac{c}{6},-\frac{a}{2}+\frac{b}{3}+\frac{c}{6}\right) .
$$

The corresponding velocities are rewritten as:

$$
\vec{v}=\left(-\frac{\dot{a}}{2}-\frac{2 \dot{b}}{3}-\frac{5 \dot{c}}{6},-\frac{\dot{a}}{2}-\frac{2 \dot{b}}{3}+\frac{\dot{c}}{6},-\frac{\dot{a}}{2}+\frac{\dot{b}}{3}+\frac{\dot{c}}{6}\right),
$$

where dot means time derivative.

For the solution of the vibration we apply Lagrange mechanics. The kinetic energy of all masses moved according the prescription to preserve the required symmetry, and which are described by the three variables is the following:

$$
T=\sum \frac{1}{2} m v_{i}^{2}=12 m\left(\frac{3}{4} \dot{a}^{2}+\dot{a} \dot{b}+\dot{b}^{2}+\frac{1}{2} \dot{a} \dot{c}+\dot{b} \dot{c}+\frac{3}{4} \dot{c}^{2}\right),
$$

and the potential energy in all atomic bonds is

$$
\begin{aligned}
U & \left.=\sum \frac{1}{2} k(\Delta a)^{2}+\sum \frac{1}{2} k(\Delta b)^{2}+\sum \frac{1}{2} k(\Delta c)^{2}\right) \\
& =6 k\left((\Delta a)^{2}+(\Delta b)^{2}+(\Delta c)^{2}\right)
\end{aligned}
$$

where $\Delta a, \Delta b$ and $\Delta c$ are the prolongations of the lengths of the edges connecting the mass points on the vertices of polytopes, i.e. the displacements in the directions of these edges. Because of $\dot{\Delta q}=\dot{q}$ we can rewrite $\Delta a=a, \Delta b=b$ and $\Delta c=c$, and these displacements can be understood as three generalized coordinates for the vertices of the polytopes.

Then the Lagrangian for the vibration preserving the symmetry is expressed by:

$$
\begin{aligned}
L= & T-U=24 m\left(\frac{3}{4} \dot{a}^{2}+\dot{a} \dot{b}+\dot{b}^{2}+\frac{1}{2} \dot{a} \dot{c}+\dot{b} \dot{c}+\frac{3}{4} \dot{c}^{2}\right) \\
& -12 k\left(a^{2}+b^{2}+c^{2}\right) .
\end{aligned}
$$

The corresponding general Euler-Lagrange equations are:

$$
\frac{d}{d t} \frac{\partial L}{\partial \dot{q}_{i}}-\frac{\partial L}{\partial q_{i}}=0
$$

In coordinates form we have three equations

$$
\begin{aligned}
m\left(\frac{3}{2} \ddot{a}+\ddot{b}+\frac{1}{2} \ddot{c}\right)+k a & =0, \\
m(\ddot{a}+2 \ddot{b}+\ddot{c})+k b & =0, \\
m\left(\frac{1}{2} \ddot{a}+\ddot{b}+\frac{3}{2} \ddot{c}\right)+k c & =0 .
\end{aligned}
$$



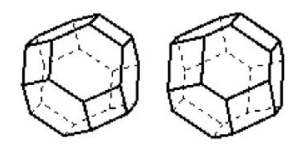

E

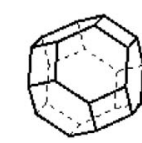

$\mathrm{E}+X_{1} \delta$

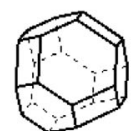

E

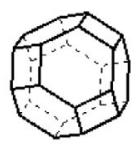

E

$\mathrm{E}+X_{2} \delta$

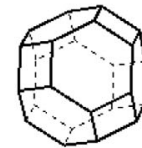

$\mathrm{E}+X_{3} \delta$

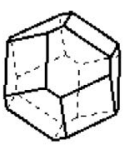

$\mathrm{E}+X_{1} 2 \delta$

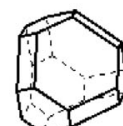

$\mathrm{E}+\mathrm{X}_{2} 2 \delta$

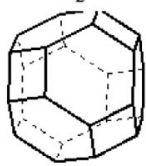

$\mathrm{E}+\mathrm{X}_{3} 2 \delta$

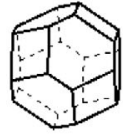

$\mathrm{E}+X_{1} \delta$

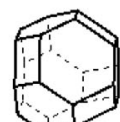

$\mathrm{E}+\mathrm{X}_{2} \delta$

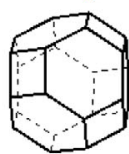

$\mathrm{E}+X_{3} \delta$

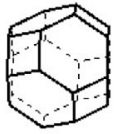

$\mathrm{E}$

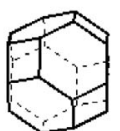

E

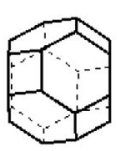

E

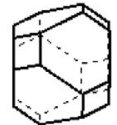

$\mathrm{E}-X_{1} \delta$

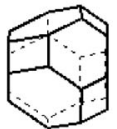

$\mathrm{E}-\mathrm{X}_{2} \delta$

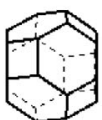

$\mathrm{E}-X_{3} \bar{\delta}$

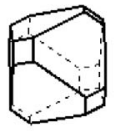

E- $X_{1} 2 \delta$

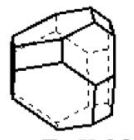

$\mathrm{E}-\mathrm{X}_{2} 2 \delta$

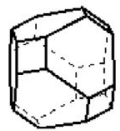

$\mathrm{E}-X_{1} \delta$

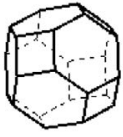

E
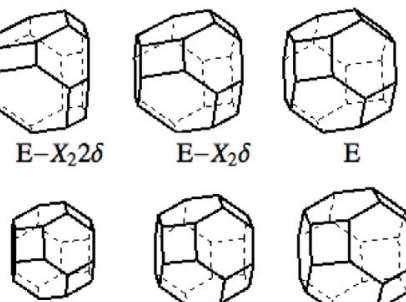

$\mathrm{E}-X_{3} 2 \delta$

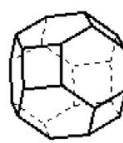

$\mathrm{E}$

Fig. 1. Imagination of vibration of polytopes of type $A_{3}$, where $E=(1,1,1), \delta=0.1$ and $X_{i}$ is eigenvector taken from Table 1 .

Table 1. Resonant frequencies for case $A_{3}$.

\begin{tabular}{ccc}
\hline Eigenvalue & Eigenvector & \\
\hline$\alpha_{1}=\frac{2+\sqrt{2}}{2}$ & $X_{1}=(1, \sqrt{2}, 1)$ & $\omega_{1}=\sqrt{\frac{k}{m}} \sqrt{2-\sqrt{2}}$ \\
$\alpha_{2}=\frac{1}{2}$ & $X_{2}=(-1,0,1)$ & $\omega_{2}=\sqrt{\frac{k}{m}} \sqrt{2}$ \\
$\alpha_{3}=\frac{2-\sqrt{2}}{2}$ & $X_{3}=(1,-\sqrt{2}, 1)$ & $\omega_{3}=\sqrt{\frac{k}{m}} \sqrt{2+\sqrt{2}}$ \\
& & $\omega_{0}=\sqrt{\frac{k}{m}} \sqrt{\frac{3}{5}}$ \\
\hline
\end{tabular}

In order to find out the modes corresponding to the vibrations with respect to the preserving symmetry, we look for the solution in the form

$$
\left(\begin{array}{l}
a \\
b \\
c
\end{array}\right)=\vec{X} \cos (\omega t+\varphi) \text {, where } \vec{X}=\left(\begin{array}{l}
A \\
B \\
C
\end{array}\right),
$$

where $\varphi$ is an arbitrary constant phase in a general solution, according to the initial conditions.

Three Euler-Lagrange equations can be written in the matrix form

$$
\mathbb{A} \frac{\mathrm{d}^{2}}{\mathrm{~d} t^{2}} \vec{X}+\mathbb{B} \vec{X}=0 \text {, i.e. }-\omega^{2} \mathbb{A} \vec{X}+\mathbb{B} \vec{X}=0
$$

The matrix $\mathbb{A}$ is

$$
\mathbb{A}=\left(\begin{array}{ccc}
3 / 4 & 1 / 2 & 1 / 4 \\
1 / 2 & 1 & 1 / 2 \\
1 / 4 & 1 / 2 & 3 / 4
\end{array}\right),
$$

and $\mathbb{B}$ is

$$
\mathbb{B}=\frac{k}{m}\left(\begin{array}{lll}
1 & 0 & 0 \\
0 & 1 & 0 \\
0 & 0 & 1
\end{array}\right) .
$$

The matrices $\mathbb{A}$ and $\mathbb{B}$ commute, therefore they have common eigenvalues and eigenvectors. Then the solutions have the following form

$$
\omega_{i}^{2} \alpha_{i} \vec{X}_{i}=\frac{k}{m} \vec{X}_{i}, \text { for } i=1,2,3,
$$

where $\alpha_{i}$ are eigenvalues, $\vec{X}_{i}$ eigenvectors and $\omega_{i}$ corresponding angular frequencies of three modes.

In all cases we can determine one more mode, corresponding to the radial oscillations. During the oscillation it preserves the shape of polytope, i.e. the central symmetry. The prolongation $(r=\Delta R)$ of the radius $(R)$ of the peripheral sphere is the generalized coordinate, because of the equidistant positions of neighbouring points. The total kinetic and potential energies (up to rest state) are:

$$
T=N \frac{1}{2} m \dot{r}^{2}, \text { and } U=\tilde{N} \frac{1}{2} k\left(2 r \sin \frac{\alpha}{2}\right)^{2},
$$

for $N$ vertices and $\tilde{N}$ edges, and where $\alpha$ is a central angle of the edge, because all of the edges are of the same length.

Euler-Lagrange equation is a harmonic oscillator equation

$$
N m \ddot{r}+4 \tilde{N} k r \sin ^{2} \frac{\alpha}{2}=0,
$$

and the corresponding mode angular frequency is $\omega_{0}^{2}=$ $4 \frac{\tilde{N} k}{N m} \sin ^{2} \frac{\alpha}{2}=\frac{\tilde{N} k}{N m} \frac{a^{2}}{R^{2}}$. For $A_{3}$ case it is $\omega_{0}^{2}=\frac{3 k}{5 m}$.

\section{Vibrations of $\mathrm{H}_{3}$ polytope}

The vertices of the icosahedral polytopes can be written in orthonormal basis when the points of the orbit are expressed as purely imaginary quaternions-icosians [8]

$$
\vec{x}=\frac{1}{\sqrt{2}}\left(\frac{\tau a}{2}+\tau b+\frac{\tau^{2} c}{2}, \frac{c}{2}, \frac{a}{2}\right) .
$$

Unlike the three crystallographic groups $A_{3}, B_{3}$ and $C_{3}$ the natural basis for the vertices of the polytopes of $H_{3}$ contains one irrationality $\tau$, which represents the golden ratio. The most naturally it is done by icosians [8], more precisely for $H_{3}$ only the 3 purely imaginary icosians.

The corresponding velocities are their time derivatives

$$
\vec{v}=\frac{1}{\sqrt{2}}\left(\frac{\tau \dot{a}}{2}+\tau \dot{b}+\frac{\tau^{2} \dot{c}}{2}, \frac{\dot{c}}{2}, \frac{\dot{a}}{2}\right) .
$$



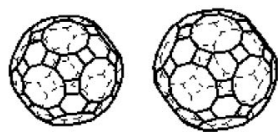

E

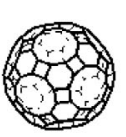

E

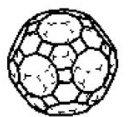

$\mathrm{E}+X_{1} \delta$

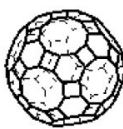

$\mathrm{E}+\mathrm{X}_{2} \delta$

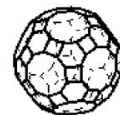

$\mathrm{E}+X_{3} \delta$

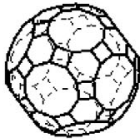

$\mathrm{E}+X_{1} 2 \delta$

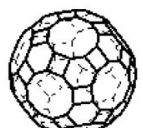

$\mathrm{E}+\mathrm{X}_{2} 2 \delta$

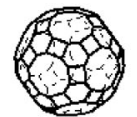

$\mathrm{E}+X_{3} 2 \delta$
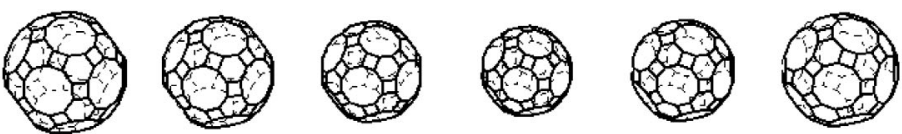

$\mathrm{E}+X_{1} \delta$
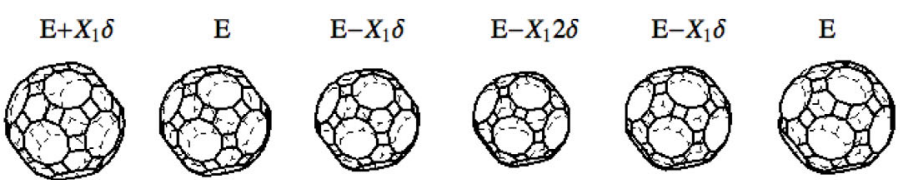

E

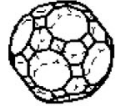

E- $X_{2} \delta$

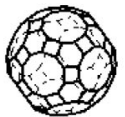

$\mathrm{E}+X_{3} \delta$

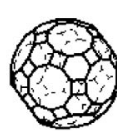

E

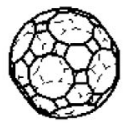

$\mathrm{E}-X_{3} \delta$

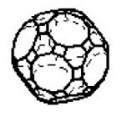

$\mathrm{E}-\mathrm{X}_{2} 2 \delta$

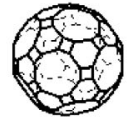

$\mathrm{E}-\mathrm{X}_{3} 2 \delta$
$\mathrm{E}-X_{2} \delta$

E

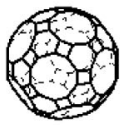

$\mathrm{E}-X_{3} \delta$

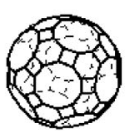

E

Fig. 2. Imagination of vibration of polytopes of type $H_{3}$, where $E=(1,1,1), \delta=0.1$ and $X_{i}$ is eigenvector taken from Table 2 .

Table 2. Resonant frequencies for case $H_{3}$.

\begin{tabular}{ccc}
\hline Eigenvalue & Eigenvector & \\
\hline$\alpha_{1}=1+\tau+\frac{1}{2} \sqrt{7+11 \tau}$ & $X_{1}=(-1+\tau, \sqrt{3-\tau}, 1)$ & $\omega_{1}=\frac{k}{m} \sqrt{4-2 \sqrt{2+\tau}}$ \\
$\alpha_{2}=\frac{1}{4}$ & $X_{2}=(\tau, 0,1)$ & $\omega_{2}=2 \frac{k}{m}$ \\
$\alpha_{3}=1+\tau-\frac{1}{2} \sqrt{7+11 \tau}$ & $X_{3}=(-1+\tau,-\sqrt{3-\tau}, 1)$ & $\omega_{3}=\frac{k}{m} \sqrt{4+2 \sqrt{2+\tau}}$ \\
& & $\omega_{0}=\sqrt{\frac{k}{m}} \sqrt{\frac{6(19-9 \tau)}{109}}$ \\
\hline
\end{tabular}

We can identify the letters $a, b$ and $c$ with the general coordinates - the displacements of masses in corresponding directions. The polytope consists of 120 vertices and 60 edges of each type $a, b$ and $c$.

The kinetic energy of all atoms is the same as a consequence of the fact that the displacement of a single point causes the displacement of all remaining points, moving with the same velocity. The total kinetic energy of movement according symmetry is equal

$$
\begin{aligned}
T= & 60 m\left(\frac{1}{4} \dot{a}^{2}(2+\tau)+\dot{a} \dot{b}(1+\tau)+\dot{b}^{2}(1+\tau)\right. \\
& \left.+\frac{1}{2} \dot{a} \dot{c}(1+2 \tau)+\dot{b} \dot{c}(1+2 \tau)+\frac{3}{4} \dot{c}^{2}(1+\tau)\right),
\end{aligned}
$$

and the potential energy in all bounds is equal

$$
U=30 k\left(a^{2}+b^{2}+c^{2}\right) .
$$

Then the Lagrangian for the vibration preserving the symmetry is calculated by:

$$
\begin{aligned}
L= & 120 m\left(\frac{1}{4} \dot{a}^{2}(2+\tau)+\dot{a} \dot{b}(1+\tau)+\dot{b}^{2}(1+\tau)\right. \\
& \left.+\frac{1}{2} \dot{a} \dot{c}(1+2 \tau)+\dot{b} \dot{c}(1+2 \tau)+\frac{3}{4} \dot{c}^{2}(1+\tau)\right) \\
& -60 k\left(a^{2}+b^{2}+c^{2}\right) .
\end{aligned}
$$

The corresponding Euler-Lagrange equations have a form

$$
\begin{gathered}
m\left(\frac{1}{2} \ddot{a}(2+\tau)+\ddot{b}(a+\tau)+\frac{1}{2} \ddot{c}(1+2 \tau)\right)+k a=0, \\
m(\ddot{a}(1+\tau)+2 \ddot{b}(1+\tau)+\ddot{c}(a+2 \tau))+k b=0, \\
m\left(\frac{1}{2} \ddot{a}(1+2 \tau)+\ddot{b}(1+2 \tau)+\frac{3}{2} \ddot{c}(1+\tau)\right)+k c=0 .
\end{gathered}
$$

The matrix $\mathbb{A}$ is then

$$
\mathbb{A}=\left(\begin{array}{ccc}
(2+\tau) / 4 & (1+\tau) / 2 & (1+2 \tau) / 4 \\
(1+\tau) / 2 & (1+\tau) & 1 / 2+\tau \\
(1+2 \tau) / 4 & 1 / 2+\tau & 3 / 4(1+\tau)
\end{array}\right),
$$

and $\mathbb{B}$ is

$$
\mathbb{B}=\frac{k}{m}\left(\begin{array}{lll}
1 & 0 & 0 \\
0 & 1 & 0 \\
0 & 0 & 1
\end{array}\right) .
$$

Because of the commutativity of both matrices there are common eigenvectors, and the solutions are

$$
\omega_{i}^{2} \alpha_{i} \vec{X}_{i}=\frac{k}{m} \vec{X}_{i}, \text { for } i=1,2,3,
$$

where $\alpha_{i}$ are eigenvalues, $\vec{X}_{i}$ eigenvectors and $\omega_{i}$ corresponding angular frequencies of three modes.

The additional mode of the prolongation $(r)$ of the radius $(R)$ of the peripheral sphere is similar as in $A_{3}$ case. The neighbour points are equidistant. The Euler-Lagrange equation is

$$
N m \ddot{r}+4 \tilde{N} k r \sin ^{2} \frac{\alpha}{2}=0,
$$

and the corresponding mode angular frequency is $\omega_{0}^{2}=$ $4 \frac{\tilde{N} k}{N m} \sin ^{2} \frac{\alpha}{2}=\frac{\tilde{N} k}{N m} \frac{a^{2}}{R^{2}}$. For $H_{3}$ case it is $\omega_{0}^{2}=\frac{6(19-9 \tau) k}{109 m}$. 

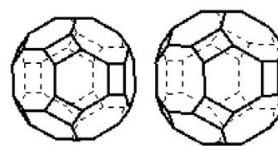

E

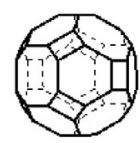

$\mathrm{E}+X_{1} \delta$

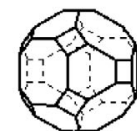

$\mathrm{E}$

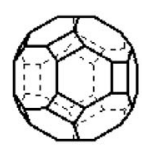

E
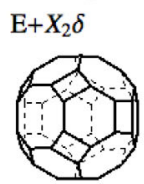

$\mathrm{E}+X_{3} \delta$

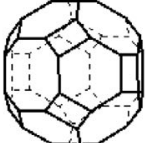

$\mathrm{E}+X_{1} 2 \delta$

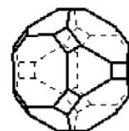

$\mathrm{E}+X_{2} 2 \delta$

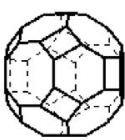

$\mathrm{E}+X_{3} 2 \delta$

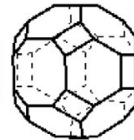

$\mathrm{E}+X_{1} \delta$

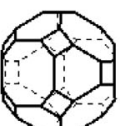

$\mathrm{E}+X_{2} \delta$

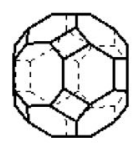

$\mathrm{E}+X_{3} \delta$
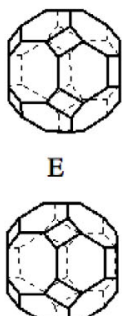

$\mathrm{E}$

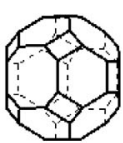

$\mathrm{E}$

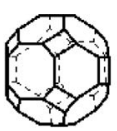

$\mathrm{E}-X_{1} \delta$

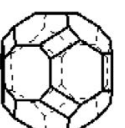

$\mathrm{E}-X_{2} \delta$

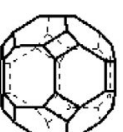

$\mathrm{E}-X_{3} \delta$
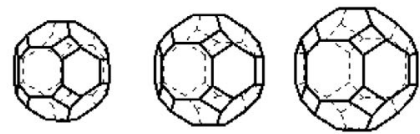

E- $X_{1} 2 \delta$

E- $X_{1} \delta$

E
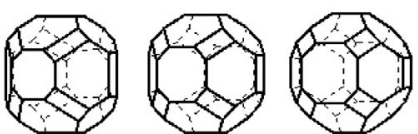

$\mathrm{E}-X_{2} 2 \delta$

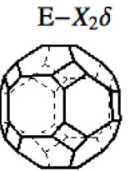

$\mathrm{E}-\boldsymbol{X}_{3} \delta$

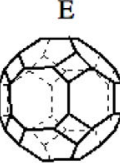

E

Fig. 3. Imagination of vibration of polytopes of type $B_{3}$, where $E=(1,1,1), \delta=0.1$ and $X_{i}$ is eigenvector taken from Table 3 .

Table 3. Resonant frequencies for case $B_{3}$.

\begin{tabular}{ccc}
\hline Eigenvalue & Eigenvector & \\
\hline$\alpha_{1}=3.13805$ & $X_{1}=(1.09477,1.84067,1)$ & $\omega_{1}=\sqrt{\frac{k}{m}} \sqrt{0.318669}$ \\
$\alpha_{2}=0.424101$ & $X_{2}=(-2.29387,0.821037,1)$ & $\omega_{2}=\sqrt{\frac{k}{m}} \sqrt{2.35793}$ \\
$\alpha_{3}=0.18785$ & $X_{3}=(0.199104,-0.661702,1)$ & $\omega_{3}=\sqrt{\frac{k}{m}} \sqrt{5.3234}$ \\
& & $\omega_{0}=\sqrt{\frac{k}{m}} \sqrt{\frac{2}{7}}$ \\
\hline
\end{tabular}

\section{Vibrations of $B_{3}$ polytope}

The octahedral polytope consists of 48 vertices and 24 edges of each type $a, b$ and $c$.

The vertices of polytope in Cartesian coordinates and the corresponding velocities have the following form:

$$
\begin{aligned}
& \vec{x}=\left(a+2 b+\frac{c}{2}, b+\frac{c}{2}, \frac{c}{2}\right), \\
& \vec{v}=\dot{\vec{x}}=\left(\dot{a}+2 \dot{b}+\frac{\dot{c}}{2}, \dot{b}+\frac{\dot{c}}{2}, \frac{\dot{c}}{2}\right) .
\end{aligned}
$$

The total kinetic and potential energies are

$$
\begin{aligned}
T & =24 m\left(\dot{a}^{2}+2 \dot{a} \dot{b}+2 \dot{b}^{2}+\dot{a} \dot{c}+2 \dot{b} \dot{c}+\frac{3}{4} \dot{c}^{2}\right), \\
U & =12 k\left(a^{2}+b^{2}+c^{2}\right) .
\end{aligned}
$$

The Lagrangian for the vibration preserving the symmetry is equal

$$
\begin{aligned}
L= & 48 m\left(\dot{a}^{2}+2 \dot{a} \dot{b}+2 \dot{b}^{2}+\dot{a} \dot{c}+2 \dot{b} \dot{c}+\frac{3}{4} \dot{c}^{2}\right) \\
& -24 k\left(a^{2}+b^{2}+c^{2}\right) .
\end{aligned}
$$

The matrix $\mathbb{A}$ has a form

$$
\mathbb{A}=\left(\begin{array}{ccc}
1 & 1 & 1 / 2 \\
1 & 2 & 1 \\
1 / 2 & 1 & 3 / 4
\end{array}\right)
$$

and $\mathbb{B}$ is

$$
\mathbb{B}=\frac{k}{m}\left(\begin{array}{lll}
1 & 0 & 0 \\
0 & 1 & 0 \\
0 & 0 & 1
\end{array}\right) .
$$

Because of the commutativity of both matrices there are common eigenvectors and the solutions are

$$
\omega_{i}^{2} \alpha_{i} \vec{X}_{i}=\frac{k}{m} \vec{X}_{i}, \text { for } i=1,2,3
$$

where $\alpha_{i}$ are eigenvalues, $\vec{X}_{i}$ eigenvectors and $\omega_{i}$ corresponding angular frequencies of three modes.

The additional mode of the prolongation $(r)$ of the radius $(R)$ of the peripheral sphere is different as in $A_{3}$ case. The neighbour points are not equidistant, because there are two different lengths of edges and so, two different central angles. The Euler-Lagrange equation is

$$
N m \ddot{r}+4 \tilde{N}_{a} k r \sin ^{2} \frac{\alpha}{2}+4 \tilde{N}_{b} k r \sin ^{2} \frac{\beta}{2}+4 \tilde{N}_{c} k r \sin ^{2} \frac{\gamma}{2}=0,
$$

and the corresponding mode angular frequency is $\omega_{0}^{2}=$ $\left(\tilde{N}_{a} \frac{a^{2}}{R^{2}}+\tilde{N}_{b} \frac{b^{2}}{R^{2}}+\tilde{N}_{c} \frac{c^{2}}{R^{2}}\right) \frac{k}{N m}$. For $B_{3}$ case when $a=b, b \neq c$ it is $\omega_{0}^{2}=\frac{2 k}{7 m}$.

\section{Vibrations of $C_{3}$ polytope}

As for $B_{3}$ case the octahedral polytopes of type $C_{3}$ have 48 vertices and 24 edges of each type $a, b$ and $c$. The vertices of a polytope and the corresponding velocities we can 


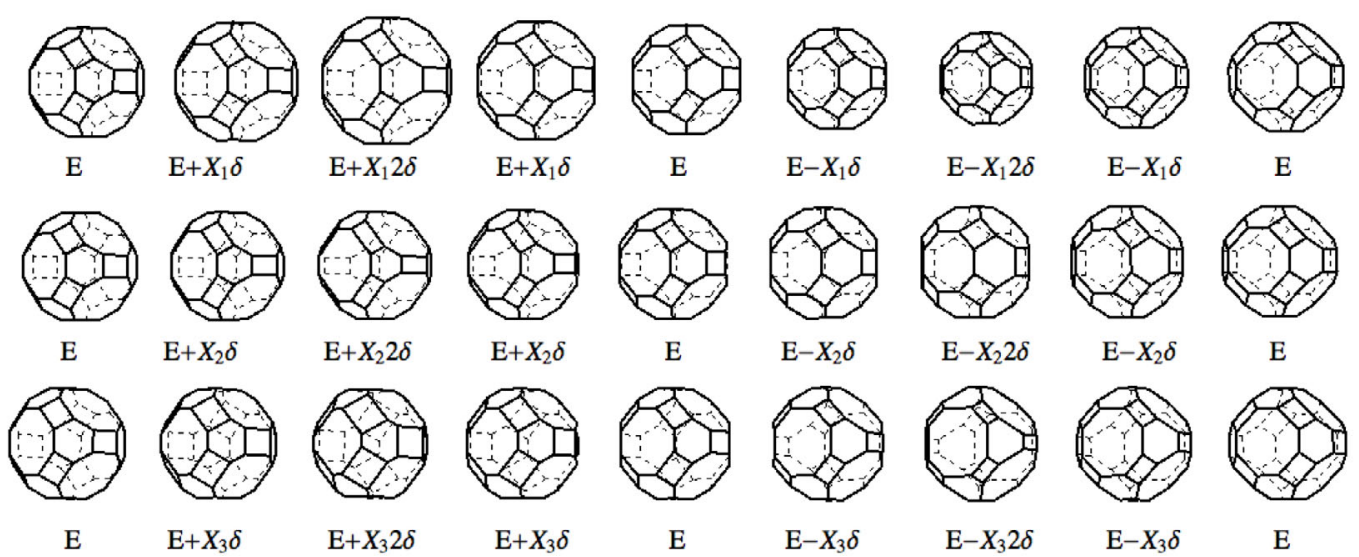

Fig. 4. Imagination of vibration of polytopes of type $C_{3}$, where $E=(1,1,1), \delta=0.1$ and $X_{i}$ is eigenvector taken from Table 4 .

Table 4. Resonant frequencies for case $C_{3}$.

\begin{tabular}{ccc}
\hline Eigenvalue & Eigenvector & \\
\hline$\alpha_{1}=2.52446$ & $X_{1}=(0.445042,0.801938,1)$ & $\omega_{1}=\sqrt{\frac{k}{m}} \sqrt{0.396125}$ \\
$\alpha_{2}=0.321552$ & $X_{2}=(-1.24698,-0.554958,1)$ & $\omega_{2}=\sqrt{\frac{k}{m}} \sqrt{3.10992}$ \\
$\alpha_{3}=0.153989$ & $X_{3}=(1.80194,-2.24698,1)$ & $\omega_{3}=\sqrt{\frac{k}{m}} \sqrt{6.49396}$ \\
& & $\omega_{0}=\sqrt{\frac{k}{m}} \sqrt{\frac{1}{6}+\sin \left(\frac{1}{2} \arccos \frac{8}{9}\right)}$ \\
\hline
\end{tabular}

write in the following form

$$
\begin{aligned}
& \vec{x}=\frac{1}{\sqrt{2}}(a+b+c, b+c, c), \\
& \vec{v}=\dot{\vec{x}}=\frac{1}{\sqrt{2}}(\dot{a}+\dot{b}+\dot{c}, \dot{b}+\dot{c}, \dot{c}) .
\end{aligned}
$$

The total kinetic energy of movement is

$$
T=24 m\left(\frac{\dot{a}^{2}}{2}+\dot{a} \dot{b}+\dot{b}^{2}+\dot{a} \dot{c}+2 \dot{b} \dot{c}+\frac{3}{2} \dot{c}^{2}\right),
$$

and the potential energy in all "springs" is

$$
U=12 k\left(a^{2}+b^{2}+c^{2}\right) .
$$

The Lagrangian for the vibration preserving the symmetry is calculated by:

$$
\begin{aligned}
L= & 48 m\left(\frac{\dot{a}^{2}}{2}+\dot{a} \dot{b}+\dot{b}^{2}+\dot{a} \dot{c}+2 \dot{b} \dot{c}+\frac{3}{2} \dot{c}^{2}\right) \\
& -24 k\left(a^{2}+b^{2}+c^{2}\right) .
\end{aligned}
$$

The matrix $\mathbb{A}$ is equal

$$
\mathbb{A}=\left(\begin{array}{ccc}
1 / 2 & 1 / 2 & 1 / 2 \\
1 / 2 & 1 & 1 \\
1 / 2 & 1 & 3 / 4
\end{array}\right)
$$

and $\mathbb{B}$ has a form

$$
\mathbb{B}=\frac{k}{m}\left(\begin{array}{lll}
1 & 0 & 0 \\
0 & 1 & 0 \\
0 & 0 & 1
\end{array}\right)
$$

Because of the commutativity of both matrices there are common eigenvectors and the solutions are

$$
\omega_{i}^{2} \alpha_{i} \vec{X}_{i}=\frac{k}{m} \vec{X}_{i}, \text { for } i=1,2,3
$$

where $\alpha_{i}$ are eigenvalues, $\vec{X}_{i}$ eigenvectors and $\omega_{i}$ corresponding angular frequencies of three modes.

The additional mode of the prolongation $(r)$ of the radius $(R)$ of the peripheral sphere is similar as in $B_{3}$ case. The neighbour points are not equidistant, because there are two different lengths of edges and so, two different central angles. The Euler-Lagrange equation is:

$N m \ddot{r}+4 \tilde{N}_{a} k r \sin ^{2} \frac{\alpha}{2}+4 \tilde{N}_{b} k r \sin ^{2} \frac{\beta}{2}+4 \tilde{N}_{c} k r \sin ^{2} \frac{\gamma}{2}=0$,

and the corresponding mode angular frequency is $\omega_{0}^{2}=$ $\left(\tilde{N}_{a} \frac{a^{2}}{R^{2}}+\tilde{N}_{b} \frac{b^{2}}{R^{2}}+\tilde{N}_{c} \frac{c^{2}}{R^{2}}\right) \frac{k}{N m}$. For $C_{3}$ case when $b=c, a \neq b$ it is $\omega_{0}^{2}=\left(\frac{1}{6}+\sin \left(\frac{1}{2} \arccos \frac{8}{9}\right)\right) \frac{k}{m}$.

\section{Concluding remarks}

The method can be used for different constant tensions of bonds in directions $a, b, c$. Then the matrices $A$ and $B$ do not commute but both are symmetric and positive definite. The algebraic solution is straightforward. The frequencies and corresponding vibrations are the solutions of the equation $\omega^{2} \mathbb{A} \vec{X}=\mathbb{B} \vec{X}$, see [9].

Each polytope with $\mathfrak{G}$ symmetry can be viewed as a sum of several polytopes with the lower symmetry than $\mathfrak{G}$. 
Techniques for reducing orbits of the group $\mathfrak{G}$ to the sum of subgroups of polytopes are known. We could imagine that vibration is caused not by a change of a single vertex or edge but a part of the original polytope which is invariant with respect to the subgroup of a symmetry group $\mathfrak{G}$. The vibration of the subgroup of polytope will cause the vibration of the entire polytope of $\mathfrak{G}$. Even more curious would be a situation when the subgroup of polytopes arise from a polytope of dimension 4 although those cannot be visualized easily. The vibration of the subgroup of polytopes then can be studied and information about the vibration of $4 \mathrm{D}$ polytopes can be inferred.

An interesting would also be studies of wave propagations along the infinite nanotubes in 3D.

\section{Author contribution statement}

All authors contributed equally to the paper.

The authors would like to express their gratitude to the Doppler Institute of the Czech Technical University for the hospitality extended to them in Prague and in Děčín, where the work was started. The authors also express thank to Jirri Tolar for fruitful discussions. G.Ch. received financial support from grant No. RVO 68407700.

\section{References}

1. D.-P. Deng, M. Widom, J. Phys. C 20, L449 (1987)

2. U. Messerschmidt, M. Bartsch, Scr. Mater. 49, 33 (2003)

3. B. Wei, in Proceedings of the Institution of Mechanical Engineers, Part C: Journal of Mechanical Engineering Science (SAGE Publishing, 2013), Vol. 2

4. M. Bodner, J. Patera, M. Szajewska, Can. J. Phys. 92, 1446 (2014)

5. M. Szajewska, Polytope contractions within Weyl group symmetries, accepted in Math. Phys. Anal. Geom. (2016)

6. G.A. Vliegenthart, G. Gompper, Biophys. J. 91, 834 (2006)

7. B. Champagne, M. Kjiri, J. Patera, R.T. Sharp, Can. J. Phys. 73, 566 (1995)

8. L. Chen, R.V. Moody, J. Patera, Fields Institute Monograph Series, in Quasicrystals and Discrete Geometry, edited by J. Patera (Amer. Math. Soc., 1998), Vol. 10, pp. $135-178$

9. H. Goldstein, C. Poole, J. Safko, Classical Mechanics, 3rd edn. (Addison-Wesley, New York, 2000)

Open Access This is an open access article distributed under the terms of the Creative Commons Attribution License (http://creativecommons.org/licenses/by/4.0), which permits unrestricted use, distribution, and reproduction in any medium, provided the original work is properly cited. 